\title{
An uncommon presentation of a common cancer
}

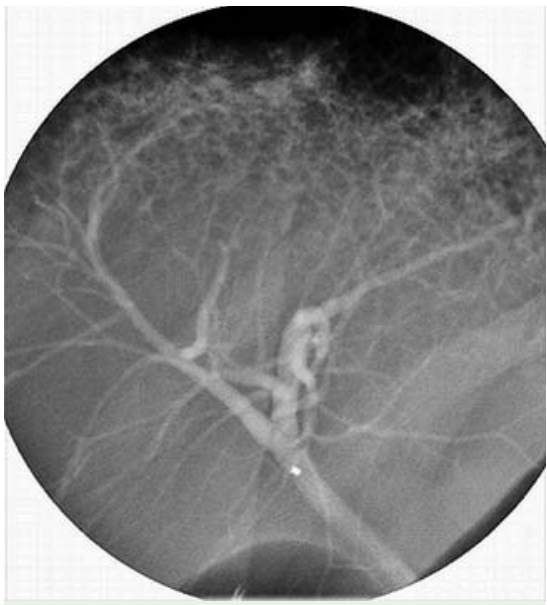

Fig. 1 Cholangiogram showing a normal appearing common bile duct.

A 67-year-old Caucasian man presenting with episodic right upper quadrant pain and weight loss of $9 \mathrm{~kg}(20 \mathrm{lb})$ was found to have elevated levels of alkaline phosphatase (180 IU/mL). An abdominal ultrasound revealed cholelithiasis with no biliary ductal dilatation. Endoscopic retrograde cholangiography (ERC) revealed a normal common bile duct (CBD) and right and left hepatic ducts ( $\bullet$ Fig. 1 ).

However, due to persistent episodes of abdominal pain, the patient was referred for a cholecystectomy. Intraoperative cholangiography showed incomplete opacification of the right hepatic duct. A subsequent computed tomography (CT) scan showed dilatation of the intrahepatic ducts in the right lobe of liver without any mass lesion. A repeat ERC showed that the actual bifurcation of the CBD was in fact lower than thought earlier ( $\bullet$ Fig. 2).

An occlusion cholangiogram showed a 2 -cm fixed lesion in the right posterior hepatic duct ( $\bullet$ Fig. 2, 3).

Brushings and biopsies were obtained from this area and were suggestive of a mucin-producing adenocarcinoma. A right hepatectomy carried out for suspected cholangiocarcinoma revealed an intrabiliary mass lesion, $4.5 \times 2.5 \mathrm{~cm}$ in size. Immunohistochemistry showed the mass to be negative for cytokeratin-7 (CK-7) and positive for CK-20 ( $\bullet$ Fig. 4, 5).

The mass was identical morphologically and immunohistochemically to the previously resected colon cancer. A diagnosis

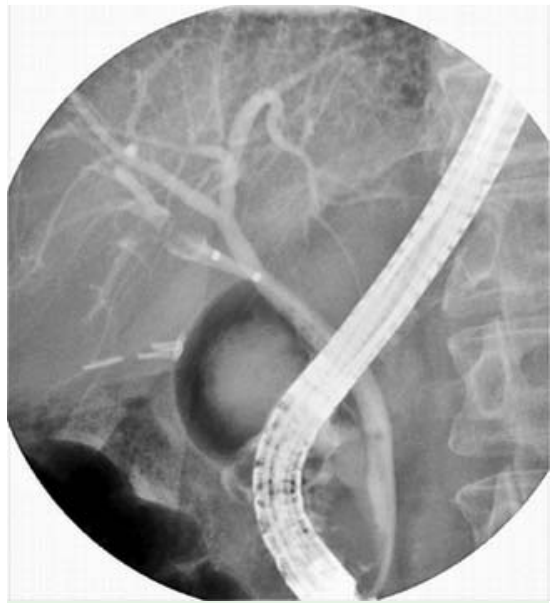

Fig. 2 Cholangiogram showing the actual bifurcation to be lower than seen in 8 Fig. 1. A filling defect is seen in the right (posterior) hepatic duct.

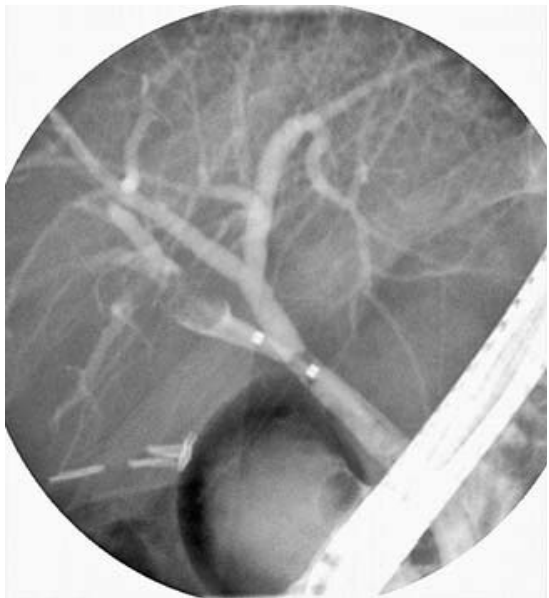

Fig. 3 Magnified view of the oval filling defect.
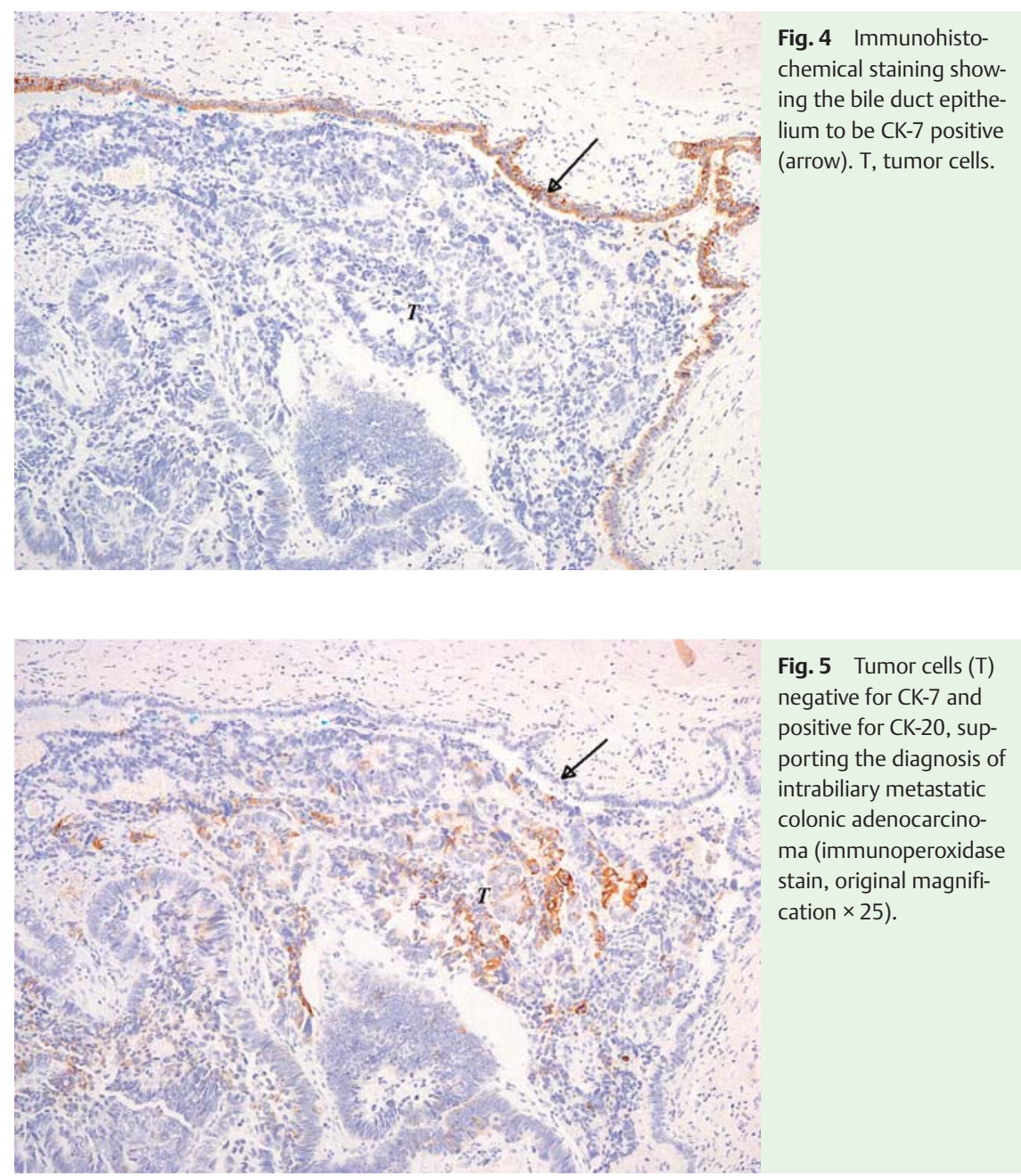
of intrabiliary metastasis from prior colon cancer was thus made and systemic chemotherapy for metastatic colon cancer was initiated. The patient went on develop recurrent metastasis in the liver and died a year later.

A history of rectosigmoid adenocarcinoma prompted immunohistochemical analysis of the resected tumor, which proved to be CK-20 positive and CK-7 negative, consistent with metastatic colon carcinoma [1]. Following hematogenous dissemination to the liver, colon cancer metastasis can occasionally demonstrate an unusual pattern of neoplastic proliferation, where it spreads along epithelial surfaces, mimicking primary biliary neoplasia [2,3]. It is important for gastroenterologists to be aware of this unusual pattern of spread of colorectal cancer. Immuno- histochemistry could help differentiate cholangiocarcinoma from metastatic colorectal cancer.

\section{Endoscopy_UCTN_Code_CCL_1AZ_2AC}

R. Tadiparthi, K. Ponnuru, R. Cherian, S. C. Mathur, A. Rastogi

Division of Gastroenterology and Hepatology, Veteran's Affairs Medical Center and University of Kansas School of Medicine, Kansas City, Missouri, USA

\section{References}

1 Chu P, Wu E, Weiss LM. Cytokeratin 7 and Cytokeratin 20 expression in epithelial neoplasms: a survey of 435 cases. Mod Pathol 2000; 13: $962-972$

2 Riopel MA, Klimstra DS, Godellas CV et al. Intrabiliary growth of metastatic colonic adenocarcinoma: a pattern of intrahepatic spread easily confused with primary biliary neoplasia of the biliary tract. Am J Surg Path 1997; 21: 1030-1036

3 Povoski SP, Klimstra DS, Brown KT et al. Recognition of intrabiliary hepatic metastases from colorectal adenocarcinoma. HPB Surg 2000; 11: 383 - 391

Bibliography

DOI $10.1055 / \mathrm{s}-0029-1243874$

Endoscopy 2010; 42: E61 -E62

(c) Georg Thieme Verlag KG Stuttgart · New York . ISSN 0013-726X

Corresponding author

\section{A. Rastogi, MD}

Gastroenterology (111)

Department of Veterans Affairs Medical Center $4801 \mathrm{E}$. Linwood Blvd

Kansas City

Missouri

USA

Amit.rastogi@med.va.gov 